\title{
Finite temperature dynamic structure function of the free Fermi gas
}

\author{
F.Mazzanti ${ }^{1}$ and A.Polls ${ }^{2}$ \\ ${ }^{1}$ Departament d'Electrònica, Enginyeria i Arquitectura La Salle, \\ Pg. Bonanova 8, Universitat Ramon Llull, \\ E-08022 Barcelona, Spain \\ 2 Departament d'Estructura i Constituents de la Matèria, \\ Diagonal 645, Universitat de Barcelona, \\ E-08028 Barcelona, Spain
}

November 21, 2018

\begin{abstract}
A detailed discussion of the coherent and incoherent dynamic structure function of the free Fermi gas at finite temperature is presented. Their behavior and evolution with the momentum transfer and the temperature is analyzed, while particular attention is paid to the way in which their relative contribution changes with respect to the $\tilde{T}=0$ case. The influence of thermal effects on the lowest order sum rules of the coherent and incoherent responses is also discussed. Finally, the scaling properties of the responses at high momentum transfer as a function of the temperature are also analyzed.
\end{abstract}

PACS: 05.30.Fk, 61.12.Bt

KEYWORDS: dynamic structure function, free Fermi gas, finite temperature 
Neutron scattering in quantum liquids at low and high momentum transfer $q$ has provided much relevant information about the role of collective excitations, the way in which singleparticle properties are affected by correlations and the nature of quantum effects in general. Theoretical models devised to explain the observed scattering data have also been developped, and the degree of sophistication achieved is such that nowadays precise understanding of many features concerning the ground state of systems like ${ }^{4} \mathrm{He},{ }^{3} \mathrm{He}$ or ${ }^{3} \mathrm{He}-{ }^{4} \mathrm{He}$ mixtures has been gained [1]. Although Path Integral Monte Carlo has supplied unique information about finite temperature effects in these systems, no particular formalisms has been capable to describe the dynamic structure function $S(q, \omega)$ at $T>0$ [2]. In a previous work we presented a detailed description of the $T=0$ dynamic structure function of the free Fermi gas, its coherent and incoherent parts and their evolution with the momentum transfer [3]. In the present letter we extend this discussion to finite temperature, focussing particularly on the influence of thermal effects on the different contributions to the response and on scaling laws.

At finite temperature, the dynamic structure function of a quantum system is proportional to the probability of coupling different states that are compatible with a momentum and energy transfer $q$ and $\omega$ when a density fluctuation moderated by the operator $\rho_{q}=\sum_{j=1}^{N} e^{i \mathbf{q} \cdot \mathbf{r}_{j}}$ takes place

$$
S(q, \omega)=\sum_{\{n, m\}} \frac{1}{\mathcal{Z}} e^{-\beta\left(E_{n}-\mu N\right)} \frac{1}{N}\left|\left\langle m\left|\rho_{q}\right| n\right\rangle\right|^{2} \delta\left(E_{m}-E_{n}-\omega\right),
$$

$\mathcal{Z}=\sum_{\{n\}} e^{-\beta\left(E_{n}-\mu N\right)}$ being the partition function of the system in the Grand Canonical ensamble, $\mu$ the chemical potential and $\beta=1 / k_{B} T$ the inverse of the temperature [4].

The dynamic structure function is also the Fourier transform of the density-density correlation factor $S(q, t)$, which can in turn be formally separated in its coherent and incoherent density responses. At finite temperature these two functions are defined as

$$
\begin{aligned}
S_{i n c}(q, t) & =\sum_{\{n\}} \frac{1}{\mathcal{Z}} e^{-\beta\left(E_{n}-\mu N\right)} \frac{1}{N} \sum_{j=1}^{N}\left\langle n\left|e^{-i \mathbf{q} \cdot \mathbf{r}_{j}} e^{i H t} e^{i \mathbf{q} \cdot \mathbf{r}_{j}} e^{-i H t}\right| n\right\rangle \\
S_{c o h}(q, t) & =\sum_{\{n\}} \frac{1}{\mathcal{Z}} e^{-\beta\left(E_{n}-\mu N\right)} \frac{1}{N} \sum_{i \neq j}^{N}\left\langle n\left|e^{-i \mathbf{q} \cdot \mathbf{r}_{i}} e^{i H t} e^{i \mathbf{q} \cdot \mathbf{r}_{j}} e^{-i H t}\right| n\right\rangle,
\end{aligned}
$$

where $H$ and $\mathbf{r}_{j}$ are the Hamiltonian and the position operator of particle $j$, respectively. Notice that in this definition of $S_{c o h}(q, \omega ; T)$ [3, 5, 6] , only those terms with $i$ strictly different from $j$ contribute to the coherent response, in contrast to the other commonly used definition where 
$S_{c o h}(q, \omega)$ is taken to be the whole scattering function given in Eq. (11) [1, 7]. Notice also that in $S_{\text {inc }}(q, t)$ correlations among different particles are indirectly accounted by correlations in the wave functions. In a free classical system where neither dynamical nor statistical correlations exist, $S_{c o h}(q, t)$ is zero and the total response equals its incoherent part. However, in realistic systems this condition is only asymptotically reached at high temperature or in the high momentum transfer limit. When $q \rightarrow \infty$, the total response of a fully correlated, infinite and isotropic system becomes mostly incoherent and conforms to the Impulse Approximation (IA). In terms of the new set of adimensional variables that will be used throughout this work $\tilde{q}=q / k_{F}, \tilde{\omega}=\omega / \epsilon_{F}$ and $\tilde{T}=T / \epsilon_{F}$ where $k_{F}$ is the $T=0$ Fermi momentum of the free Fermi gas and $\epsilon_{F}=k_{F}^{2} / 2 m$, the adimensional IA reads

$$
\tilde{S}_{I A}(\tilde{q}, \tilde{\omega} ; \tilde{T}) \equiv \epsilon_{F} S_{I A}(q, \omega ; T)=\frac{3}{4 \pi} \int d \tilde{\mathbf{k}} n(\tilde{k}) \delta\left[(\tilde{\mathbf{k}}+\tilde{\mathbf{q}})^{2}-\tilde{k}^{2}-\tilde{\omega}\right]=\frac{3}{4 \tilde{q}} \int_{|\tilde{Y}|}^{\infty} \tilde{k} n(\tilde{k}) d \tilde{k}
$$

where $\tilde{Y}=(\tilde{\omega} / \tilde{q}-\tilde{q}) / 2$ is the West scaling variable $[8$ and $n(\tilde{k})$ is the finite temperature occupation probability of each single-particle state of definite momentum. Corrections to the IA are moderated by the interatomic potential, so in a free system the IA exactly coincides with $S_{\text {inc }}(\tilde{q}, \tilde{\omega})$ at any value of $\tilde{q}$ and $\tilde{\omega}[5]$.

The dynamic structure function is also related to the imaginary part of the dynamic sus-

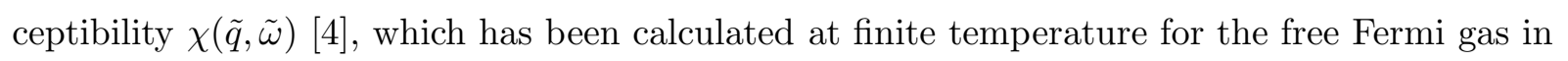
ref. [9] and reads

$$
\tilde{S}(\tilde{q}, \tilde{\omega} ; \tilde{T})=\frac{3 \tilde{T}}{8 \tilde{q}} \frac{1}{\left(1-e^{-\tilde{\omega} / \tilde{T}}\right)} \ln \left(\frac{1+z e^{-\frac{1}{4 \tilde{T}}\left(\frac{\tilde{\omega}}{\tilde{q}}-\tilde{q}\right)^{2}}}{1+z e^{-\frac{1}{4 \tilde{T}}\left(\frac{\tilde{\omega}}{\tilde{q}}+\tilde{q}\right)^{2}}}\right)
$$

where $z=e^{\tilde{\mu} / \tilde{T}}$ is the adimensional fugacity at temperature $\tilde{T}$ and $\tilde{\mu}=\mu / \epsilon_{F}$ is the adimensional chemical potential. This last quantity can be determined from the particle normalization condition

$$
\frac{2}{3} \tilde{T}^{-3 / 2}=\int_{0}^{\infty} \frac{\epsilon^{1 / 2} d \epsilon}{z^{-1} e^{\epsilon}+1}
$$

which only depends on the temperature and therefore yields a density and mass independent chemical potential. Notice that at finite $\tilde{T}$, a system can deexcite giving some energy to the probe; as a consequence the response is also deffined at negative energies. Actually in thermodynamical equilibrium, the response satisfies detailed balance

$$
\tilde{S}(\tilde{q},-\tilde{\omega} ; \tilde{T})=e^{-\tilde{\omega} / \tilde{T}} \tilde{S}(\tilde{q}, \tilde{\omega} ; \tilde{T})
$$


as can be straightforwardly checked in Eq. (司).

The adimensional incoherent response of a free system can be computed in the Impulse Approximation

$$
\tilde{S}_{i n c}(\tilde{q}, \tilde{\omega} ; \tilde{T})=\frac{3 \tilde{T}}{8 \tilde{q}} \ln \left(1+z e^{-\frac{1}{4 \tilde{T}}\left(\frac{\tilde{\omega}}{\tilde{q}}-\tilde{q}\right)^{2}}\right)
$$

while the coherent response $\tilde{S}_{c o h}(\tilde{q}, \tilde{\omega} ; \tilde{T})$ is the difference between (5) and (8). Notice that for the free Fermi gas $\tilde{S}(\tilde{q}, \tilde{\omega} ; \tilde{T})$ can also be obtained from the well known relation

$$
\tilde{S}(\tilde{q}, \tilde{\omega} ; \tilde{T})=\frac{3}{4 \pi} \int d \tilde{\mathbf{k}} n(\tilde{k})[1-n(\tilde{\mathbf{k}}+\tilde{\mathbf{q}})] \delta\left((\tilde{\mathbf{k}}+\tilde{\mathbf{q}})^{2}-\tilde{k}^{2}-\tilde{\omega}\right)
$$

where the finite temperature momentum distribution is

$$
n(\tilde{k})=\frac{1}{z^{-1} e^{\tilde{k}^{2} / \tilde{T}}+1},
$$

which yields occupation numbers between 0 and 1 . A direct consequence of the previous deffinitions is that $\tilde{S}_{i n c}(\tilde{q}, \tilde{\omega} ; \tilde{T})$ is positive, has no nodes and is symmetric around the point $\tilde{\omega}=\tilde{q}^{2}$ where it shows a peak. In much the same way, $\tilde{S}(\tilde{q}, \tilde{\omega} ; \tilde{T})$ is always lower or equal to $\tilde{S}_{i n c}(\tilde{q}, \tilde{\omega} ; \tilde{T})$, and therefore $\tilde{S}_{c o h}(\tilde{q}, \tilde{\omega} ; \tilde{T})$ is negative and does not change sign. Furthermore, the coherent response is an even function of the energy and has its minimum at $\tilde{\omega}=0$. Finally, $\tilde{S}(\tilde{q}, \tilde{\omega} ; \tilde{T})$ and $\tilde{S}_{i n c}(\tilde{q}, \tilde{\omega} ; \tilde{T})$ are positive functions satisfying $\tilde{S}(\tilde{q}, \tilde{\omega} ; \tilde{T}) \leq \tilde{S}_{i n c}(\tilde{q}, \tilde{\omega} ; \tilde{T})$, therefore the absolute value of $\tilde{S}_{c o h}(\tilde{q}, \tilde{\omega} ; \tilde{T})$ is always lower or equal to $\tilde{S}_{i n c}(\tilde{q}, \tilde{\omega} ; \tilde{T})$.

The $\tilde{q}=0.1$ and $\tilde{q}=1$ total, coherent and incoherent responses of the free Fermi gas are compared at $\tilde{T}=1$ and $\tilde{T}=0$ in Fig. (1), where the zero temperature responses are taken from ref. 3]

$$
\tilde{S}(\tilde{q}, \tilde{\omega} ; 0)=\left\{\begin{array}{ll}
\frac{3 \tilde{\omega}}{8 \tilde{q}} & \text { if } 2 \tilde{q}-\tilde{q}^{2} \geq \tilde{\omega} \geq 0 \\
\frac{3}{8 \tilde{q}}\left[1-\frac{1}{4}\left(\frac{\tilde{\omega}}{\tilde{q}}-\tilde{q}\right)^{2}\right] & \text { if } 2 \tilde{q}+\tilde{q}^{2} \geq \tilde{\omega} \geq 2 \tilde{q}-\tilde{q}^{2} \\
\frac{3}{8 \tilde{q}}\left[1-\frac{1}{4}\left(\frac{\tilde{\omega}}{\tilde{q}}-\tilde{q}\right)^{2}\right] & \text { if } \tilde{q}^{2}+2 \tilde{q} \geq \tilde{\omega} \geq \tilde{q}^{2}-2 \tilde{q} \\
0 \text { otherwise } &
\end{array} \quad \tilde{q} \geq 2 .\right.
$$

and

$$
\tilde{S}_{i n c}(\tilde{q}, \tilde{\omega} ; 0)=\frac{3}{8 \tilde{q}}\left[1-\left(\frac{\tilde{\omega}}{\tilde{q}}-\tilde{q}\right)^{2}\right] .
$$

At the momenta considered, both the coherent and the incoherent responses are finite and contribute to the total dynamic structure function. As it can be seen from the figure, temperature 
effects noticeably quench $\tilde{S}_{i n c}(\tilde{q}, \tilde{\omega} ; \tilde{T})$ and $\tilde{S}_{c o h}(\tilde{q}, \tilde{\omega} ; \tilde{T})$ even though the net effect on the latter is stronger, thus leading to a total response that is more incoherent at finite temperature than at $\tilde{T}=0$. This effect turns out to be particularly relevant at low $\tilde{q}$ 's, where the reduction in $\tilde{S}_{c o h}(\tilde{q}, \tilde{\omega} ; \tilde{T})$ is large compared to the reduction in $\tilde{S}_{i n c}(\tilde{q}, \tilde{\omega} ; \tilde{T})$ and the total response becomes strongly enhanced with respect to $\tilde{S}(\tilde{q}, \tilde{\omega} ; 0)$. The quenching of the incoherent response may be understood recalling that at finite temperature single-particle states are occupied according to Eq. (10), and while particle number conservation requires the second $\tilde{k}$-weighted moment of $n(\tilde{k})$ not to change with temperature, the way in which the different states above and below the Fermi level are filled at $\tilde{T}>0$ is such that the total contribution of $\tilde{k} n(\tilde{k})$ at fixed $\tilde{\omega}$ is lower than at $\tilde{T}=0$. Much more significant is the quenching suffered by the coherent response at low energies, which is due to the simultaneous action of two effects. On one hand and as commented above, $\tilde{S}_{\text {coh }}(\tilde{q}, \tilde{\omega} ; \tilde{T})$ is always smaller than $\tilde{S}_{i n c}(\tilde{q}, \tilde{\omega} ; \tilde{T})$ in absolute value, and the latter is at finite temperature lower than at $\tilde{T}=0$. On the other, at finite temperature and low momentum transfer the occupation probability of the states at which particles transite when the system is given a net momentum $\tilde{\mathbf{q}}$ is not 1 , and hence the effect of Pauli correlations is weakened compared to the $\tilde{T}=0$ case.

As it can also be seen in the figure, the energy interval covered by the three responses at finite temperature is larger than the range at $\tilde{T}=0$. According to eqs. (蛋) and (10), $\tilde{S}_{\text {inc }}(\tilde{q}, \tilde{\omega} ; \tilde{T})$ contributes at all energies (both positive and negative) because in the IA the allowed transitions have energy $\tilde{\omega}=(\tilde{\mathbf{k}}+\tilde{\mathbf{q}})^{2}-\tilde{k}^{2}$, and at finite temperature $n(\tilde{k})$ may extend up to infinity. In contrast, the $\tilde{T}=0$ occupation is restricted to states with $\tilde{k} \leq 1$ and so the energy range covered by $\tilde{S}_{i n c}(\tilde{q}, \tilde{\omega} ; 0)$ at fixed $\tilde{q}$ reduces to the interval $\tilde{\omega} \in\left(\tilde{q}^{2}-2 \tilde{q}, \tilde{q}^{2}+2 \tilde{q}\right)$. Consequently, thermal excitations promote particles to states above the Fermi level and these contribute to the large energy tails of the response, which therefore become enhanced with respect to the $\tilde{T}=0$ case.

It is also important to notice that the incoherent response takes into account the occupation of the initial states but disregards any possible constrain on the occupation of the final states at which the excited particles transite. As symmetry requirements restrict the set of particle configurations that can be realized in the final state, the coherent response subtracts from the incoherent one the contribution of all those transitions that are actually forbidden by the Pauli exclussion principle, thus leading to a total response in which correlations in both the initial and 
the final states are properly treated. That is the reason why at fixed temperature and momentum transfer $\tilde{S}_{c o h}(\tilde{q}, \tilde{\omega} ; \tilde{T})$ is negative and lower or equal to $\tilde{S}_{i n c}(\tilde{q}, \tilde{\omega} ; \tilde{T})$ in absolute value. Finally, as $\tilde{S}_{c o h}(\tilde{q}, \tilde{\omega} ; \tilde{T})$ subtracts strength from $\tilde{S}_{i n c}(\tilde{q}, \tilde{\omega} ; \tilde{T})$, and the latter extends over an energy interval larger than the range covered at $\tilde{T}=0$, the same happens to $\tilde{S}_{c o h}(\tilde{q}, \tilde{\omega} ; \tilde{T})$. In this sense, the tails of the finite temperature coherent response are enhanced when compared to $\tilde{S}_{c o h}(\tilde{q}, \tilde{\omega} ; 0)$.

However, and as it is shown in Fig. (2), a completely different situation is found when the transferred momentum $\tilde{q}$ is equal or larger than 2 . At $\tilde{q}>2, \tilde{S}_{c o h}(\tilde{q}, \tilde{\omega} ; 0)$ cancels and the total response becomes entirely incoherent, while at finite temprature neither $\tilde{S}_{i n c}(\tilde{q}, \tilde{\omega} ; \tilde{T})$ nor $\tilde{S}_{c o h}(\tilde{q}, \tilde{\omega} ; \tilde{T})$ vanish and hence $\tilde{S}(\tilde{q}, \tilde{\omega} ; \tilde{T})$ has contributions from both functions. In this sense, therefore, temperature acts as a source of coherentness, even though the total response is clearly dominated by $\tilde{S}_{i n c}(\tilde{q}, \tilde{\omega} ; \tilde{T})$.

The evolution with $\tilde{T}$ of the total response and its coherent and incoherent parts is reported in figure (3) for $\tilde{q}=1$ and four different values of the adimensional temperature. As at high $\tilde{T}$ almost all particles have been promoted outside the Fermi sphere and the mean occupation of every single-particle state is low, statistical effects are drastically weakened and the system reaches the classical limit. In this case and due to the absence of correlations, the response becomes completely incoherent and conforms to the IA computed from a Maxwellian momentum distribution

$$
\tilde{S}_{c l}(\tilde{q}, \tilde{\omega} ; \tilde{T})=\frac{1}{2 \tilde{q} \sqrt{\pi \tilde{T}}} e^{-\frac{1}{4 \tilde{T}}\left(\frac{\tilde{\omega}}{\tilde{q}}-\tilde{q}\right)^{2}},
$$

a result that can be easily derived from eq.(12) using the asymptotic expression of the chemical potential [10]

$$
\tilde{\mu}(\tilde{T} \rightarrow \infty) \rightarrow \tilde{T} \ln \left(\frac{4}{3 \pi^{1 / 2} \tilde{T}^{3 / 2}}\right)
$$

As it can be seen from the figure, the low temperature coherent, incoherent and total responses depart from their classical prediction, i.e. $\tilde{S}_{\text {coh }}(\tilde{q}, \tilde{\omega} ; \tilde{T})=0$ and $\tilde{S}(\tilde{q}, \tilde{\omega} ; \tilde{T})=\tilde{S}_{\text {inc }}(\tilde{q}, \tilde{\omega} ; \tilde{T}) \equiv$ $\tilde{S}_{c l}(\tilde{q}, \tilde{\omega} ; \tilde{T})$. At finite temperature, $\tilde{S}_{c o h}(\tilde{q}, \tilde{\omega} ; \tilde{T})$ is negative and $\tilde{S}_{i n c}(\tilde{q}, \tilde{\omega} ; \tilde{T})$ is positive, but when $\tilde{T}$ is raised the former goes to zero while the latter approaches the classical limit, a fact that brings the total response closer to $\tilde{S}_{c l}(\tilde{q}, \tilde{\omega} ; \tilde{T})$ but at a slower rate. Notice, however, that the way in which the classical limit is approached depends on the value of the momentum transfer. At $\tilde{q}<2$ the contribution of the coherent response is maximal at $\tilde{T}=0$ and decreases with increasing $\tilde{T}$. At $\tilde{q}>2 \tilde{S}_{\text {coh }}(\tilde{q}, \tilde{\omega} ; \tilde{T})$ is finite at intermediate temperatures but vanishes at $\tilde{T}=0$ 
and $\tilde{T} \rightarrow \infty$. In this way, temperature brings incoherentness to the response when $\tilde{q}<2$ and coherentness when $\tilde{q}>2$. The presence of coherent effects is also reflected in the shape of the total response, as when $\tilde{S}_{c o h}(\tilde{q}, \tilde{\omega} ; \tilde{T})$ is zero $\tilde{S}(\tilde{q}, \tilde{\omega} ; \tilde{T})$ equals $\tilde{S}_{\text {inc }}(\tilde{q}, \tilde{\omega} ; \tilde{T})$ and thus the total response becomes maximal and symmetric around $\tilde{\omega}=\tilde{q}^{2}$. Out of this limit, $\tilde{S}(\tilde{q}, \tilde{\omega} ; \tilde{T})$ is asymmetric and peaks at some positive energy larger than $\tilde{q}^{2}$, a direct consequence of $\tilde{S}(\tilde{q}, \tilde{\omega} ; \tilde{T})$ being the sum of $\tilde{S}_{\text {inc }}(\tilde{q}, \tilde{\omega} ; \tilde{T})$ and $\tilde{S}_{c o h}(\tilde{q}, \tilde{\omega} ; \tilde{T})$ while the latter is negative and minimal at $\tilde{\omega}=0$. In this sense, both the asymmetry of the response and the shift of its maximum to slightly larger energies is a clear signature of the presence of coherent contributions.

The role played by coherent and incoherent effects at finite temperature can also be analyzed in terms of sum rules, which are energy weighted integrals of the different responses

$$
\begin{aligned}
& \tilde{m}_{i n c, c o h}^{(\alpha)}(\tilde{q} ; \tilde{T}) \equiv \int_{-\infty}^{\infty} \tilde{\omega}^{\alpha} \tilde{S}_{i n c, c o h}(\tilde{q}, \tilde{\omega} ; \tilde{T}) d \tilde{\omega} \\
& \tilde{m}^{(\alpha)}(\tilde{q} ; \tilde{T}) \equiv \tilde{m}_{i n c}^{(\alpha)}(\tilde{q} ; \tilde{T})+\tilde{m}_{c o h}^{(\alpha)}(\tilde{q} ; \tilde{T})=\int_{-\infty}^{\infty} \tilde{\omega}^{\alpha} \tilde{S}(\tilde{q}, \tilde{\omega} ; \tilde{T}) d \tilde{\omega}
\end{aligned}
$$

Up to the first two orders, these read

$$
\begin{array}{ccc}
\tilde{m}_{\text {inc }}^{(0)}(\tilde{q} ; \tilde{T})=1 & \tilde{m}_{\text {coh }}^{(0)}(\tilde{q} ; \tilde{T})=\tilde{S}(\tilde{q} ; \tilde{T})-1 & \tilde{m}^{(0)}(\tilde{q} ; \tilde{T})=\tilde{S}(\tilde{q}, \tilde{T}) \\
\tilde{m}_{\text {inc }}^{(1)}(\tilde{q} ; \tilde{T})=\tilde{q}^{2} & \tilde{m}_{\text {coh }}^{(1)}(\tilde{q} ; \tilde{T})=0 & \tilde{m}^{(1)}(\tilde{q} ; \tilde{T})=\tilde{q}^{2}
\end{array}
$$

where $\tilde{S}(\tilde{q} ; \tilde{T})$ is the finite temperature static structure factor. The $\tilde{m}^{(1)}(\tilde{q} ; \tilde{T})$ sum rules do not yield particular information about the behavior of the free Fermi gas because they are known to be satisfied by both correlated and uncorrelated homogeneous and isotropic systems. On the other hand, the static structure factor is a positive defined quantity that in the current case turns out to be smaller or equal to 1 , as can be inferred from the properties of $n(\tilde{k})$ and the deffinition of $\tilde{S}(\tilde{q}, \tilde{\omega} ; \tilde{T})$ in eq.(9). The analysis of such an integrated quantity brings information about how coherent and incoherent effects globally affect the total response, in contrast to the previous local analysis in which the contribution of $\tilde{S}_{i n c}(\tilde{q}, \tilde{\omega} ; \tilde{T})$ and $\tilde{S}_{c o h}(\tilde{q}, \tilde{\omega} ; \tilde{T})$ at each $\tilde{\omega}$ was discussed.

$\tilde{S}(\tilde{q} ; \tilde{T})$ can be numerically evaluated and is shown for several temperatures in Fig.(4). As it can be seen, the finite temperature and the $\tilde{T}=0$ static structure factors are different. At low momentum, $\tilde{m}_{c o h}^{(0)}(\tilde{q} ; \tilde{T})$ is closer to 0 than $\tilde{m}_{c o h}^{(0)}(\tilde{q} ; 0)$, thus showing that $\tilde{S}(\tilde{q}, \tilde{\omega} ; \tilde{T})$ is globally less coherent that $\tilde{S}(\tilde{q}, \tilde{\omega} ; 0)$. In this way, the visible local reduction in the strength of $\tilde{S}_{\text {coh }}(\tilde{q}, \tilde{\omega} ; \tilde{T})$ 
at low $\tilde{q}$ 's and $\tilde{\omega}$ 's originates an overall global reduction of coherentness in the total response (see Fig.(1)). However, at high $\tilde{q}$ the opposite situation is found. At $\tilde{T}=0$ the static structure factor is 1 for all $\tilde{q}>2$ while at finite temperature this value is only asymptotically reached when $\tilde{q} \rightarrow \infty$. In this sense, therefore, and in contrast to what happens at low momentum transfer, thermal effects induce coherent contributions at high $\tilde{q}$. Notice, however, that depending on the value of $\tilde{q}$ this effect may not be easily appreciated in the plots. This is because at high momentum transfer the global enhancement of coherentness in the total response is mainly due to a strong broadening of $\tilde{S}_{c o h}(\tilde{q}, \tilde{\omega} ; \tilde{T})$ over the whole energy axis rather than to a local increase of strength at low energies.

One of the most interesting features of the density response of a system of interacting particles is the scaling behaviour, i.e. in the high momentum transfer limit the response becomes mainly incoherent and is driven by the West scaling variable $\tilde{Y}$. In order to put in evidence the scaling one introduces the adimensional Compton profile

$$
\tilde{J}(\tilde{q}, \tilde{Y} ; \tilde{T})=2 \tilde{q} \tilde{S}(\tilde{q}, \tilde{\omega} ; \tilde{T})
$$

In this context scaling means that $\tilde{J}(\tilde{q}, \tilde{Y} ; \tilde{T})$ does not depend on $\tilde{q}$. In the free case, the only source of correlations is the Pauli principle and therefore as stated before the incoherent response and the impulse approximation coincide. The adimensional Compton profile, associated to the incoherent response, at finite $\tilde{T}$ reads

$$
\tilde{J}_{I A}(\tilde{Y} ; \tilde{T})=\frac{3}{4} \tilde{T} \ln \left(1+e^{-\frac{\left(\tilde{Y}^{2}-\tilde{\mu}\right)}{\tilde{T}}}\right)
$$

where variable $q$ has been omitted for being superflous. Notice that this adimensional expression depends only on $\tilde{Y}$ and $\tilde{T}$, thus being mass and density independent. $\tilde{J}_{I A}(\tilde{q} ; \tilde{T})$ is also a symmetric function of $\tilde{Y}$. At $\tilde{T}=0$ this expresion reduces to

$$
\tilde{J}_{I A}(\tilde{Y} ; 0)=\frac{3}{4}\left(1-\tilde{Y}^{2}\right)
$$

defined in the interval $-1 \leq \tilde{Y} \leq 1$. On the other hand, when $\tilde{T} \rightarrow \infty$ one obtains the Compton profile associated to the classical response

$$
\tilde{J}_{c l}(\tilde{Y} ; \tilde{T})=\frac{1}{\sqrt{\pi \tilde{T}}} e^{-\tilde{Y}^{2} / \tilde{T}} .
$$

At zero temperature and for $\tilde{q} \geq 2$, the Pauli principle has no effect and so the total response is completly incoherent and scales according to Eq. (19). When the temperature incresases, the 
incoherent part still scales but due to the Pauli principle the coherent part of the response is not zero and so the scaling property of the total response is lost.

Of course, at fixed temperature the scaling regime is recovered when $\tilde{q}$ increases. This fact is illustrated in Fig.(5) where the Compton profile associated to the full response $\tilde{J}(\tilde{q}, \tilde{Y} ; \tilde{T})$ is reported at $\tilde{T}=1$ and for different values of $\tilde{q}$. Curves corresponding to $\tilde{q}=2$ and $\tilde{q}=3$ would coincide at $\tilde{T}=0$, and thus their difference is a measure of how thermal effects break scaling. Already at $\tilde{q}=3$ the asymptotic behaviour has almost been reached and the Compton profile is very well approximated by $\tilde{J}_{I A}(\tilde{Y} ; \tilde{T})$.

Another interesting point is to study how at fixed $\tilde{q}$ the response approaches the classical limit $\tilde{J}_{c l}(\tilde{Y} ; \tilde{T})$ when temperature increases. However, as $\tilde{J}_{c l}(\tilde{Y} ; \tilde{T})$ itself depends on $\tilde{T}$, it is useful to eliminate this dependence by introducing a new variable $\tilde{Y}_{T}=\tilde{Y} / \tilde{T}^{1 / 2}$ and a new Compton profile $\tilde{\jmath}(\tilde{q}, \tilde{Y} ; \tilde{T})$

$$
\tilde{\jmath}\left(\tilde{q}, \tilde{Y}_{T} ; \tilde{T}\right) \equiv \tilde{T}^{1 / 2} \tilde{J}(\tilde{q}, \tilde{Y} ; \tilde{T})
$$

With this new definition,

$$
\tilde{\jmath}_{c l}\left(\tilde{Y}_{T}\right)=\frac{1}{\sqrt{\pi}} e^{-\tilde{Y}_{T}^{2}}
$$

which is an universal function valid for all free and classical systems. In Fig. (6a) $\tilde{\jmath}\left(\tilde{q}, \tilde{Y}_{T} ; \tilde{T}\right)$ is plotted at $\tilde{q}=1$ as a function of $\tilde{T}$. At low temperatures $\tilde{\jmath}\left(\tilde{q}, \tilde{Y}_{T} ; \tilde{T}\right)$ is remarkably different from $\tilde{\jmath}_{c l}\left(\tilde{Y}_{T}\right)$ and not symmetric in $\tilde{Y}_{T}$. When $\tilde{T}$ increases, $\tilde{\jmath}\left(\tilde{q}, \tilde{Y}_{T} ; \tilde{T}\right)$ approaches $\tilde{\jmath}_{c l}\left(\tilde{Y}_{T}\right)$. In Fig. (6b) the same comparison is shown for the incoherent part of the response $\tilde{\jmath}_{I A}\left(\tilde{Y}_{T} ; \tilde{T}\right)$. In this case the curves are independent of $\tilde{q}$ because the latter scales. In addition, $\tilde{\jmath}_{I A}\left(\tilde{Y}_{T} ; \tilde{T}\right)$ is a symmetric function of $\tilde{Y}_{T}$ that approaches $\tilde{\jmath}_{c l}\left(\tilde{Y}_{T}\right)$ when $\tilde{T}$ increases. Notice that these definitions of $\tilde{\jmath}$ s is appropriate only to study the limit of high temperature.

In summary, the dynamic structure function of the free Fermi gas has been formally separated in its coherent and incoherent parts inorder to analyze the influence of thermal effects in the total response. The relative contribution of the coherent and incoherent responses turns out to depend on the momentum transfer. At low $\tilde{q}$ the main effect produced by the temperature is to reduce coherentness from the response. On the other hand, at high $\tilde{q}$, thermal effects act as a source of coherentness. As a consequence, temperature breaks the scaling behaviour characteristic of the high $\tilde{q}$ response at $\tilde{T}=0$. However, scaling is recovered in the $\tilde{T} \rightarrow \infty$ limit, where the total response approaches the classical limit. Despite of the simplicity of the 
system considered, the present analysis can give insight in understanding the behaviour of the response of very dilute interacting Fermi systems.

This work has been partially supported by grants DGICYT (Spain) PB95-1249 and the program SGR98-11 from Generalitat de Catalunya. 


\section{References}

[1] H.R. Glyde, Excitations in Solid and Liquid Helium, Clarendon Press, Oxford (1994) and references therein.

[2] D.M. Ceperley, Rev.Mod.Phys. 67, 279 (1995).

[3] F. Mazzanti, A. Polls and J. Boronat, Phys.Lett. A220, 251 (1996).

[4] D. Pines, and P. Nozières, The Theory of Quantum Liquids, Vol.I, Addison-Wesley (1989).

[5] Momentum Distributions edited by P.E.Sokol, R.N. Silver and J.W. Clark. Plenum Press, New York (1989).

[6] H.A. Gersch, L.J. Rodriguez, and P.N. Smith, Phys. Rev. A5,1547(1972).

[7] S.W. Lovesey, Theory of Neutron Scattering from Condensed Matter, Clarendon Press, Oxford (1984).

[8] J.B. West, Phys.Rep. 18C, 263 (1975).

[9] F.C. Khanna and H.R. Glyde, Can.J.Phys. 54, 648 (1976).

[10] R.K. Pathria, Statistical Mechanics, Pergamon Press, New York (1972). 


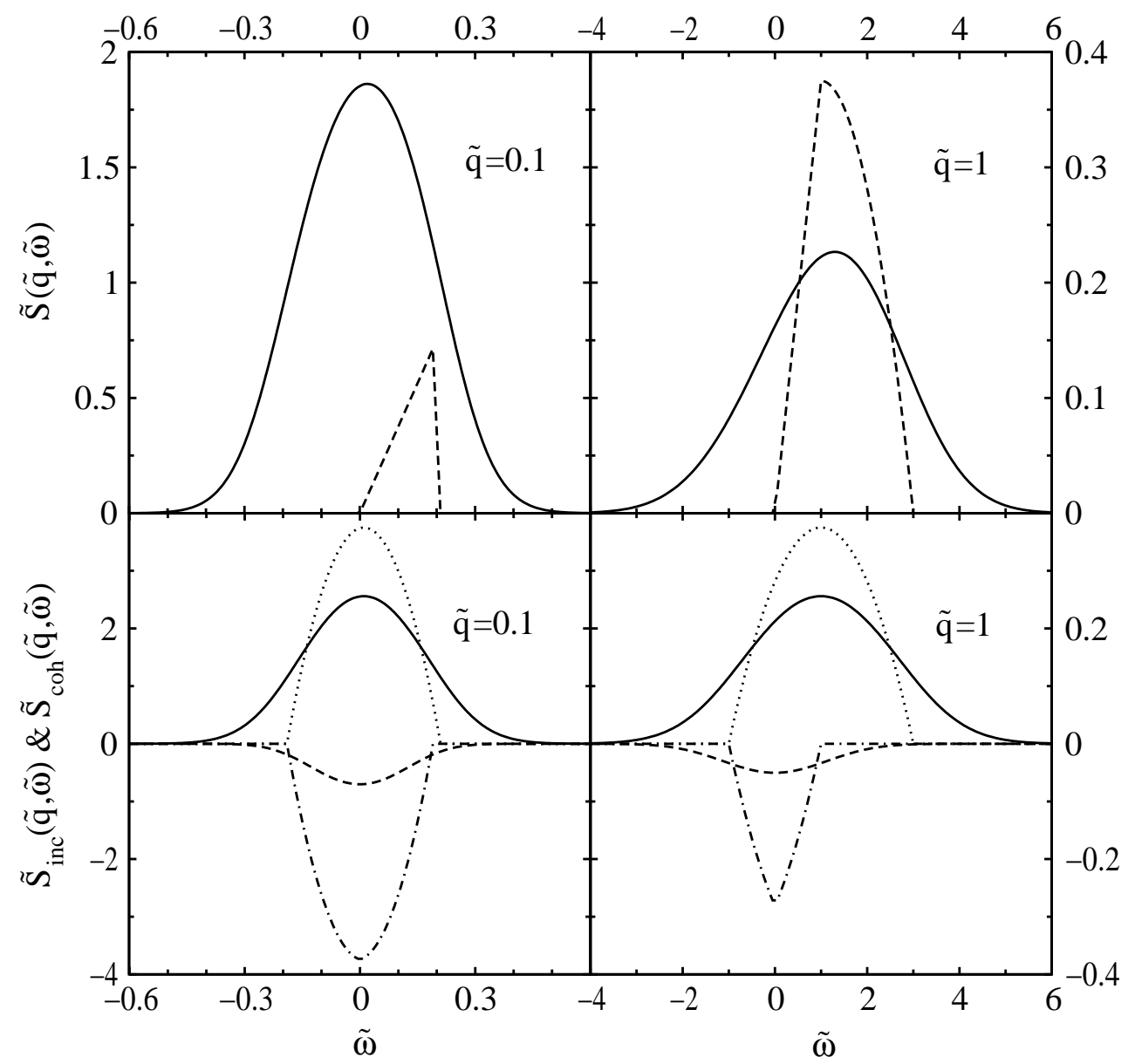

Figure 1: Total response and its coherent and incoherent parts at $\tilde{T}=0$ and $\tilde{T}=1$. Upper plots: total response at $\tilde{T}=1$ (solid line) and $\tilde{T}=0$ (dashed lines) at $\tilde{q}=0.1$ and $\tilde{q}=1$. Lower plots: incoherent responses at $\tilde{T}=1$ (solid line) and at $\tilde{T}=0$ (dotted lines), and coherent responses at $\tilde{T}=1$ (dashed line) and $\tilde{T}=0$ (dot-dashed line). 


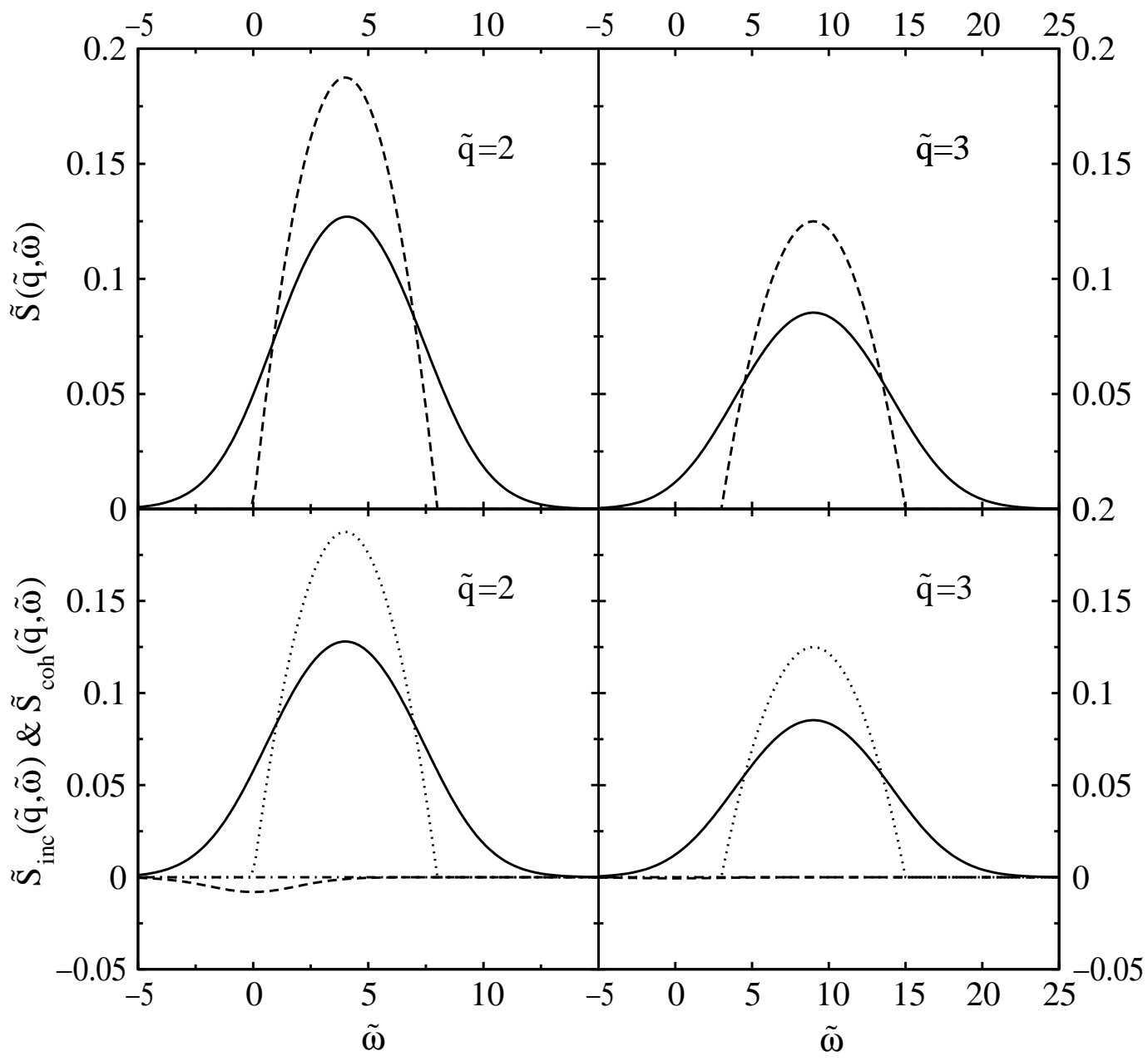

Figure 2: Total response and its coherent and incoherent parts at $\tilde{T}=0$ and $\tilde{T}=1$, for $\tilde{q}=2$ and $\tilde{q}=3$, with the same notation used in Fig. (11). 


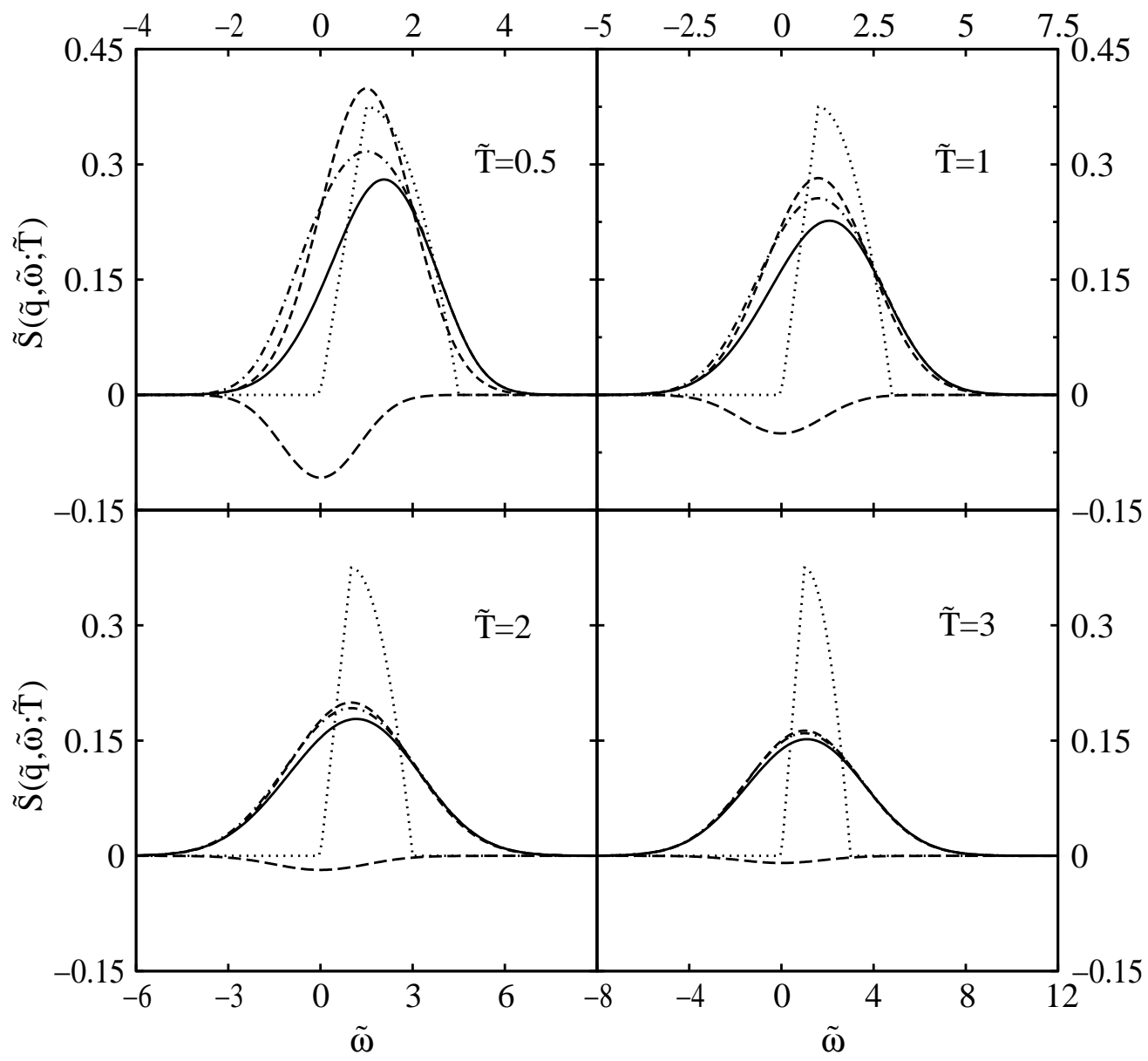

Figure 3: Different responses at several temperatures. Solid line: total response at finite temperature, dotted line: total response at $\tilde{T}=0$, dot-dashed line: incoherent response at $\tilde{T} \neq 0$, long-dashed line: coherent response at $\tilde{T} \neq 0$, dashed line: clasical response at $\tilde{T} \neq 0$. 


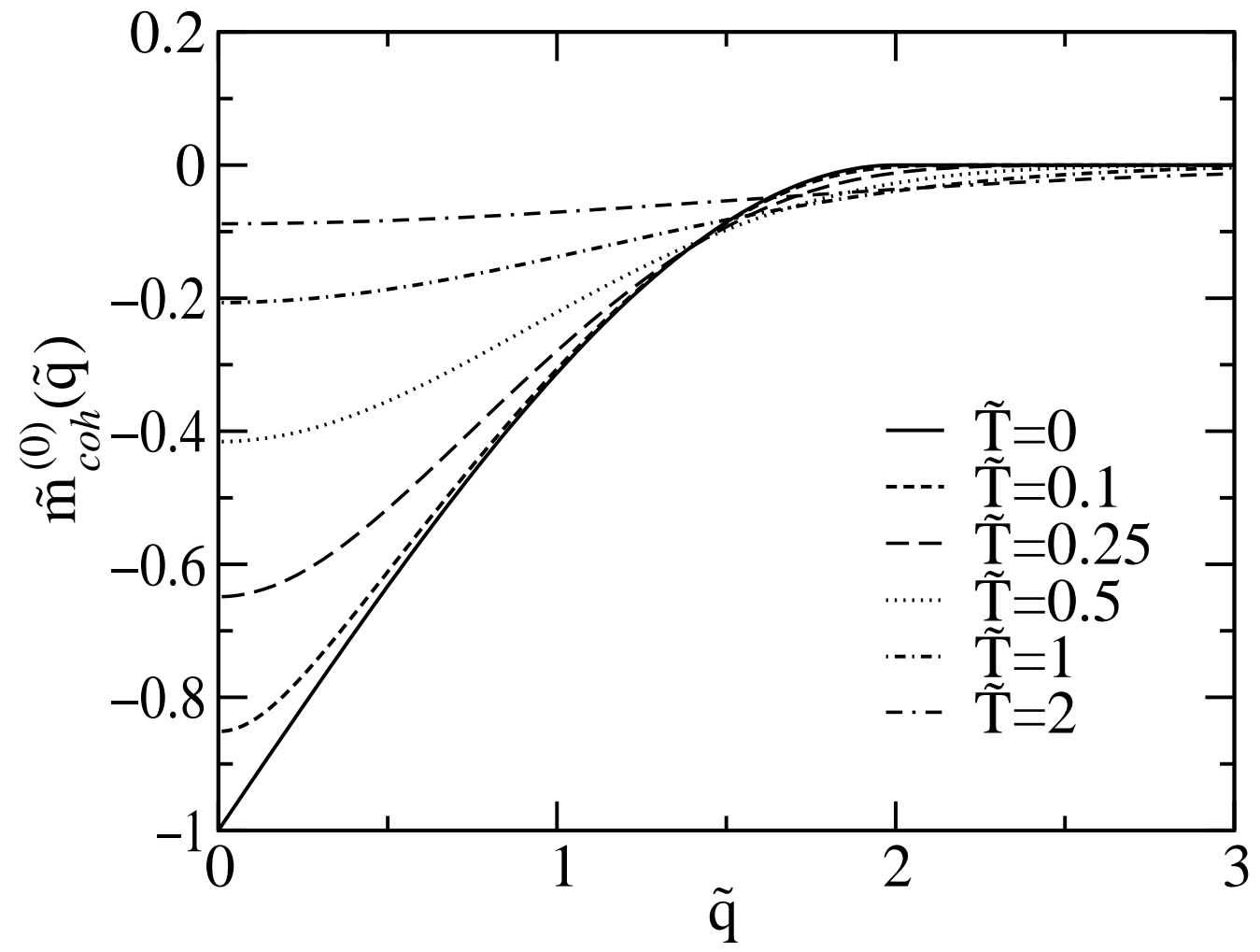

Figure 4: Momentum dependence of the zero order sum rule of the coherent response at different temperatures. 


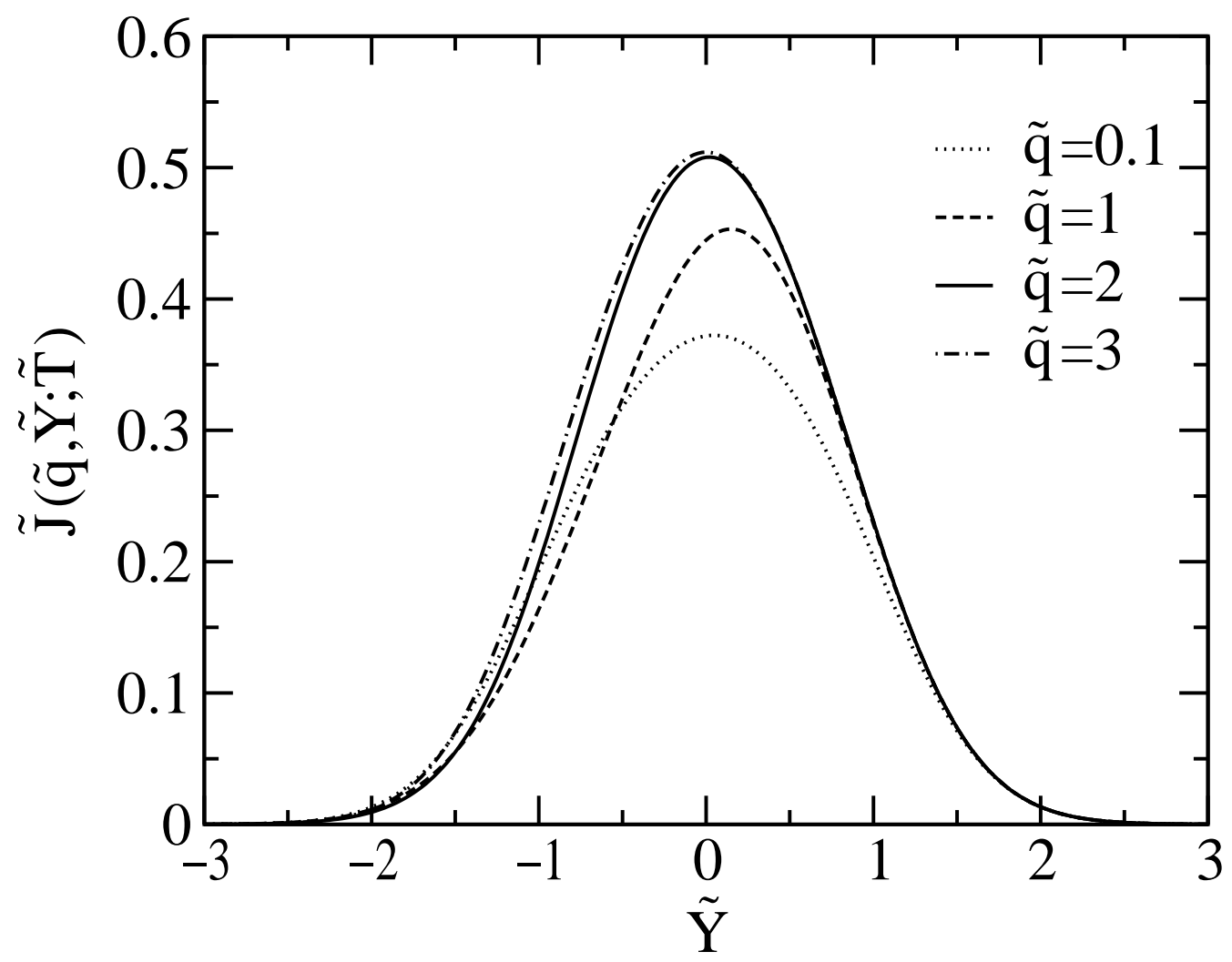

Figure 5: Finite temperature Compton profile of the total response at $\tilde{T}=1$ and several values of the momentum transfer $\tilde{q}$. 


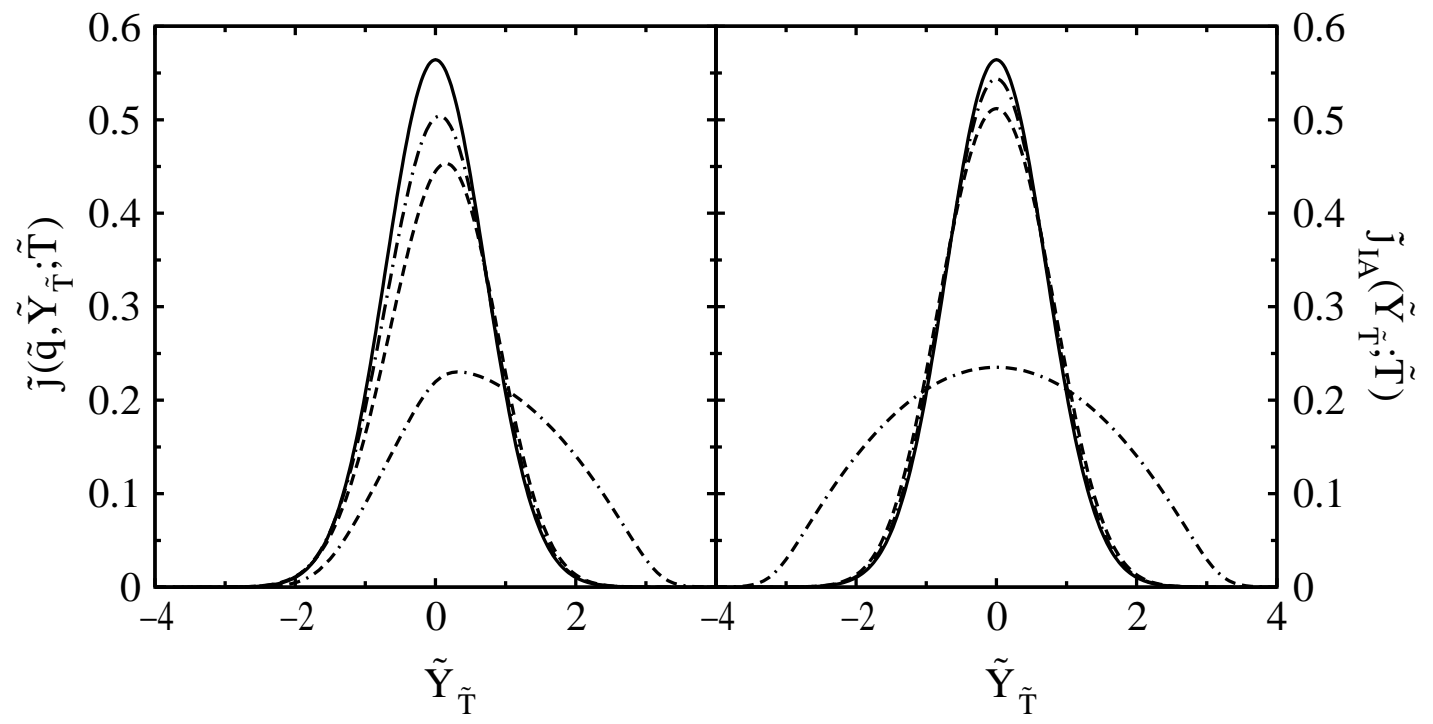

Figure 6: (a) $\tilde{\jmath}\left(\tilde{q}, \tilde{Y}_{T} ; \tilde{T}\right)$ and $\tilde{\jmath}_{I A}\left(\tilde{Y}_{T} ; \tilde{T}\right)$ at $\tilde{q}=1$ for several temperatures. Solid line: universal $\tilde{\jmath}_{c l}\left(\tilde{Y}_{T}\right)$ (see Eq. (22)); dot-dashed line: $\tilde{T}=2$; dashed line: $\tilde{T}=1$, and dot-dashed-dashed: $\tilde{T}=0.1$. 